\title{
Interactions of different Janus particles with passive tracers
}

\author{
Purnesh Chattopadhyay, Juliane Simmchen
}

March 2019

\section{Introduction}

Differently from laboratory settings, in which artificial swimmers are mostly observed, biological active particles like sperm cells, algae, bacteria or motor proteins often encounter other particles or different obstacles. For the case of bacteria as purely hydrodynamic pusher, the interaction with a wall was explained by hydrodynamic interactions of disturbance flows back-coupling with the interfacial thin layer giving rise to trapping behaviours.[1] This fostered the interest of the interactions of active Janus colloids with their surroundings, e.g. how active $\mathrm{Pt} @ \mathrm{SiO}_{2}$ particles interact with substrates and walls, finding a constant angle of 0 degrees considering the motion vector of the particles.[2]

For this particular type of phoretic swimmers also the chemical interactions have shown to be of significant relevance, mainly their strength or chemical nature.[3] Uspal discusses two possible interaction mechanisms: either via the wall induced changes in the solute gradients that finally affect the phoretic slip, or via the confinement of the hydrodynamic flow.[3] In a refined continuum model Uspal and collaborators showed that the orientation found in experiments could be achieved by a combination of both, when including also higher order effects and gravity.[2] Contemporarily, Das et al. published similar findings.[4] Both studies find and elucidate the orientation of active Janus particles on flat solid walls, causing a straightening of the paths which is sometimes also referred to as quenching of rotational diffusion. In the literature it has been found, that sometimes large objects and walls are causing very similar behaviours, $[5,6]$

However, there is a large number experimental findings, which show the different interactions in very different systems. Some of the older literature focus on the transport of passive cargoes. $[7,8,9]$ On the other hand, biological swimmers, as well as electrophoretic motors seem not to show significant 'stickiness' for passive tracers. $[10,11]$ However, even for rather different motion mechanisms, such as a spinodal decomposition of the critical mixtures, interesting interactions have been reported, for example a slightly repulsive interaction has been reported parenthetically by Buttinoni et al.[12] and later used to induce formation of colloidal clusters, as well as their compression and surface melting.[13] Active passive interactions for metallodielectric Janus particles and dielectric passives in $\mathrm{AC}$ electric fields have been extensively studied. Here, the interactions are due to dielectricphoretic interplays induced by the different polarizability compared to medium.[14, $15,16,17,18]$ Here, not only a strong dependence on the frequency was observed [19], but also the crucial importance of the material combinations, compare [15] and [16]. For light driven systems even a certain tuneability by variation of fuel and light wavelength has been reported.[20, 21] Excellent reviews on the general topic have been published over the last years. [22, 23] 
While in experiments many studies on active passive systems have been discussed, in theory the majority of interactions that have been discussed are on interactions where both partners are active. [24, 25, 26, 27, 28] The theoretical treatment has become a focus more recently. [29, 30, 31] Here, we want to expand the report on tuneable phoretic interactions. Previously, we had kept the swimmer body fixed and varied fuel and light source.[20] In this report we fix the fuel to hydrogen peroxide and change the species that favours its degradation, i.e. the 'catalytic' half on the Janus particles. To do so, many materials are known. Our selection includes copper and silver, which present interesting effects besides the well known platinum.

\section{Material and Methods}

All reagents, including $2 \mu \mathrm{m} \mathrm{SiO}_{2}$ particles and $\mathrm{H}_{2} \mathrm{O}_{2}$, were purchased from Sigma and were of analytical grade and used as received without further treatment. Milli-Q water was used for preparing aqueous solutions.

Monolayers of $2 \mu \mathrm{m} \mathrm{SiO}_{2}$ particles were prepared by Langmuir-Blodgett deposition on cleaned glass covers, using a suspension of particles $5 \mathrm{wt} \%$ in a mixture of ethanol and chloroform (1:4). Subsequently, the particles were coated with a $15 \mathrm{~nm}$ metal layer by thermal deposition $(\mathrm{Ag}, \mathrm{Cu})$ or sputtering $(\mathrm{Pt})$. To remove the particles from the cover slides short ultrasound pulses were sufficient.

The motion and interaction of the particles were observed under a Zeiss light microscope. The particles were placed on plasma cleaned glass slides, delimited by Grace Bio-Lab's SecureSeal imaging spacers. Videos were recorded at a frame rate of $40 \mathrm{fps}$ in 1 and $5 \%$ of peroxide in miliQ water using a Zeiss camera. The particles are tracked using ImageJ. Over 50 particles are studied for each condition and then their instantaneous velocities are averaged. In the active passive interaction cases, the concentration of passives was of $0.05 \mathrm{wt} \%$ of $2 \mu \mathrm{m} \mathrm{SiO}_{2}$. The motion of only active particles are studied and for $\mathrm{Pt}$ and $\mathrm{Cu}$ the whole time interval of each trajectory is used for calculation of speed values. For Ag only the first 120 frames are averaged, since the speed decays strongly during the process of reaction. For the value in presence of passive particles the speed over all particles, within a raft or not, is given.

\section{Results}

\section{1 $\mathrm{Pt} @ \mathrm{SiO}_{2}$ Janus - attractive interactions}

Using well known $\mathrm{Pt} @ \mathrm{SiO}_{2}$ Janus particles the motion direction we observe is away from the cap, as frequently reported. $[32,33]$ In this case all the particles that are hit by the active particle in forward direction, i.e. with the inert cap, are slightly attracted and incorporated in the formation of a raft, similarly to the light driven $\mathrm{Cu} @ \mathrm{TiO}_{2}$ motors in peroxide and UV light.[20] It is regularly observed, that the raft formation slows down, as has been also theoretically reported. [31] Subsequently, the motion reaches a complete stop of motility, which is not related to a stop of activity, since the raft prevails. 


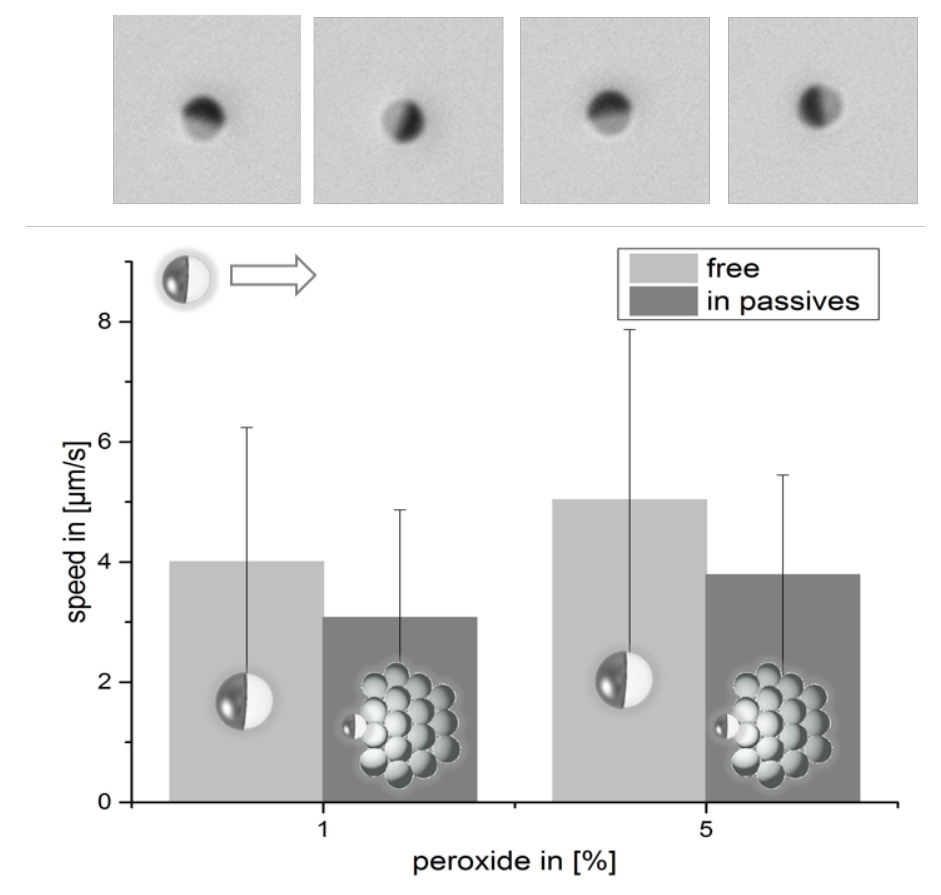

Figure 1: orientation and speed of platinum catalyzed Janus particles.

Looking at the trajectory of an active particle in close vicinity of passives, it becomes obvious that the interaction length is very short. Particles are not visibly attracted towards the active particle, only at a distance of less than a particle radius an interaction becomes visible. In Figure 2 this results in normal Brownian motion for all the passive particles that are not in direct contact with the active Janus swimmer. Only the particles that are directly hit by the active swimmer and being incorporated into the raft are bound and show reduced Brownian motion.

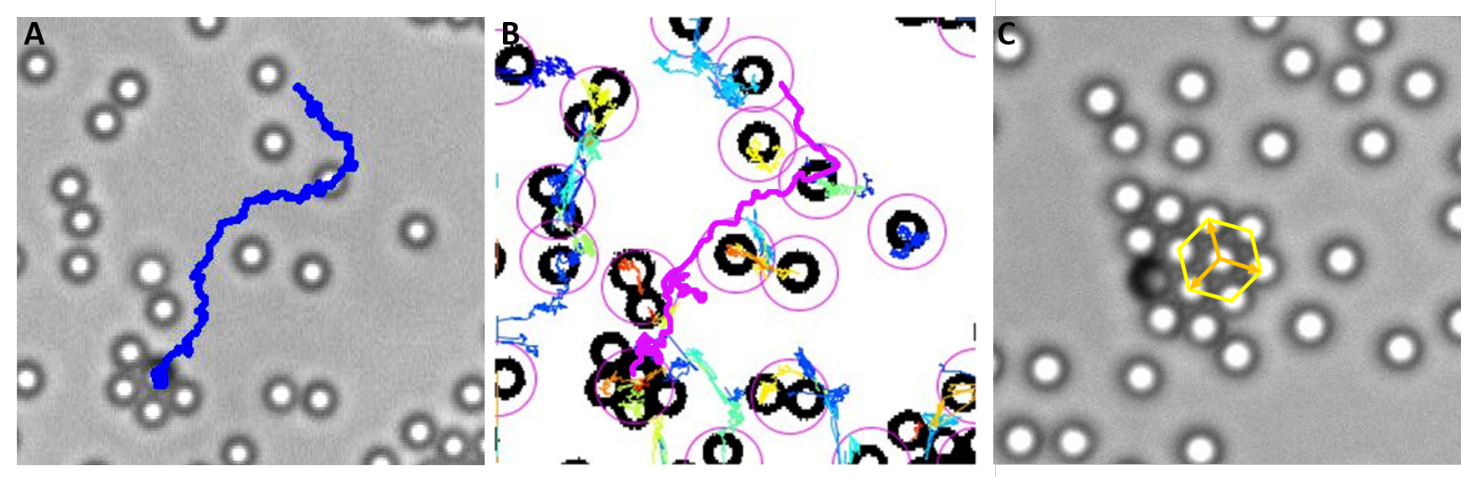

Figure 2: trajectory and raft formation of platinum Janus particles. 
Figure 3 shows the time dependence of the formed rafts. Formation starts soon after the addition of peroxide, depending on the random trajectories of Janus particles and their encounters with passives. Nearly all rafts show an asymmetric hexagonal assembly of passive particles around the active, as can be seen in Figure 2 C). Formed rafts rarely disassemble before the fuel runs out, in Figure 3 the durability of the rafts during a 10 minute experiments is shown to maintain comparability with the experiments in Figure 6 and 9, but much longer lasting assemblies can be achieved, considering that we work in a reservoir of fuel.

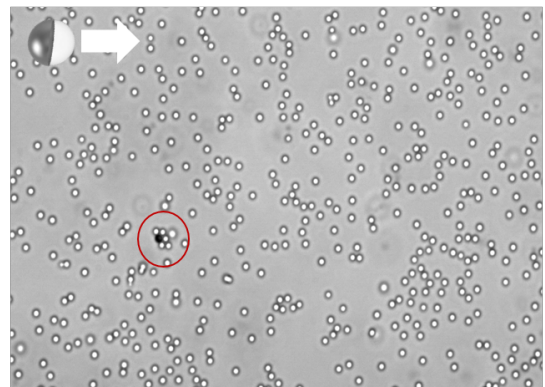

$1 \mathrm{~min}$ after peroxide addition

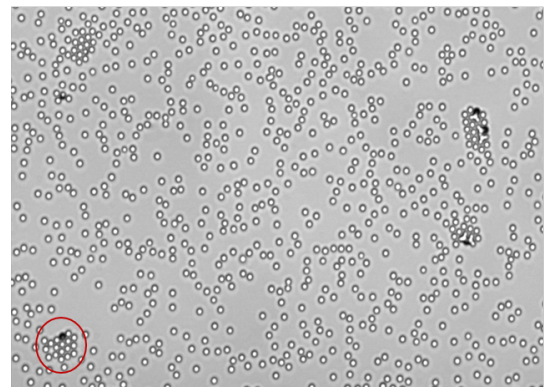

+5 mins

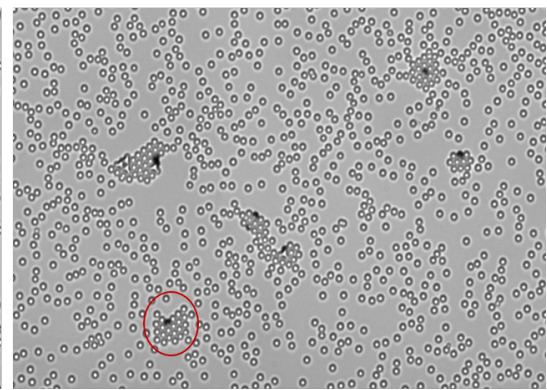

+10 mins

Figure 3: time dependence of raft formation around platinum Janus particles.

\section{$3.2 \quad \mathrm{Cu} @ \mathrm{SiO}_{2}$ Janus - repulsive interactions}

Copper capped Janus particles also orient on the bottom surface with the vector of motion parallel to the substrate. However, motion direction is towards the cap, i.e. in opposite direction compared to the platinum Janus particles. Speeds are within the same order of magnitude as platinum particles.

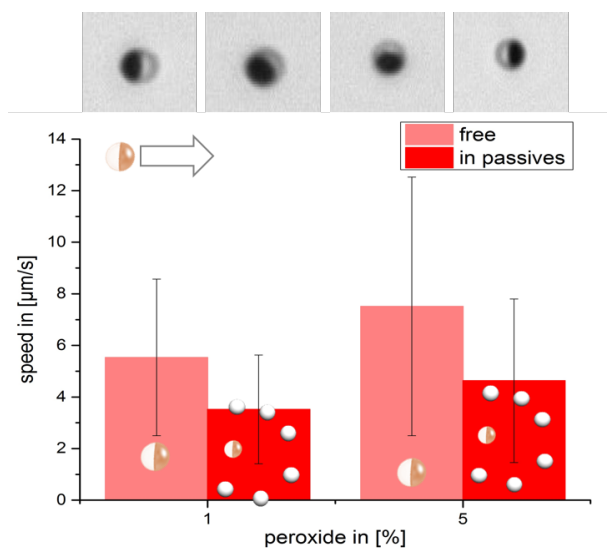

Figure 4: orientation and speed of copper catalyzed Janus particles.

In presence of passive particles, the interactions differ completely from platinum particles, mov- 
ing into opposite direction. Once the copper Janus particle approaches a passive, at a distance of several particle radii the passives start being influenced by the gradient surrounding the Janus particles and are repelled by it. This leads to a slow, steady motion away from the motion direction of the Janus particle, causing exclusion zones around the copper Janus particles. It should be noticed, that this interaction is clearly more long ranged than the attractive interactions we observe for platinum particles, similarly to the use of a copper layer on $\mathrm{TiO}_{2}$ particles in peroxide and visible light.[20] This observation might be identical, considering that the $\mathrm{TiO}_{2}$ should just behave as an inert particle in visible light, due to its larger band gap.
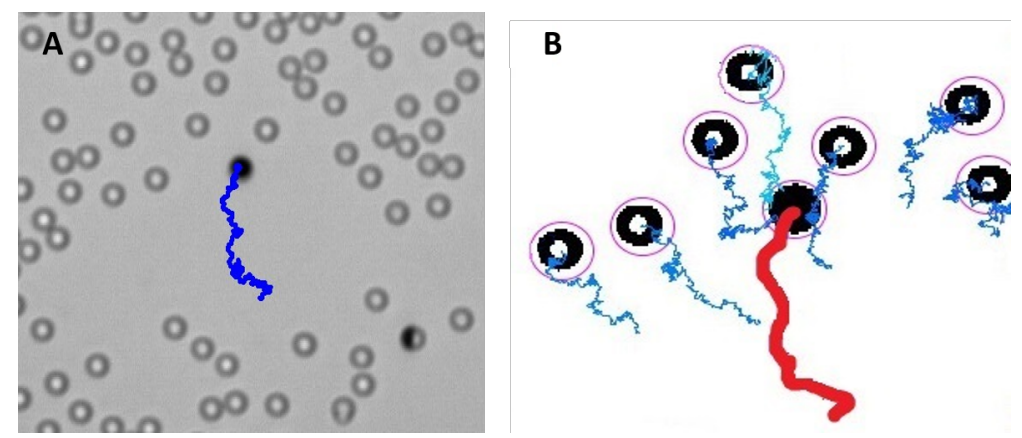

Figure 5: trajectory and interaction with passives of copper Janus particles.

Here, the speed of motion decays faster compared to platinum Janus particles, which is accompanied by a quicker decay in the formation of exclusion zones around the copper particles. When initially strong exclusion zones of about 5-15 particle radii are observed, these are less pronounced but still re-traceable after 5 minutes. The state after 10 minutes shows, that most exclusion zones have been repopulated by the normal Brownian motion of passives, only in few cases any difference is still noticeable.

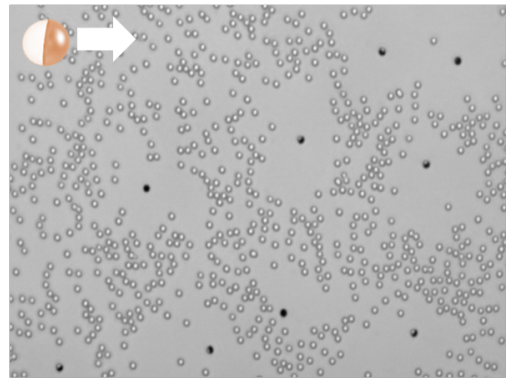

$1 \mathrm{~min}$ after peroxide addition

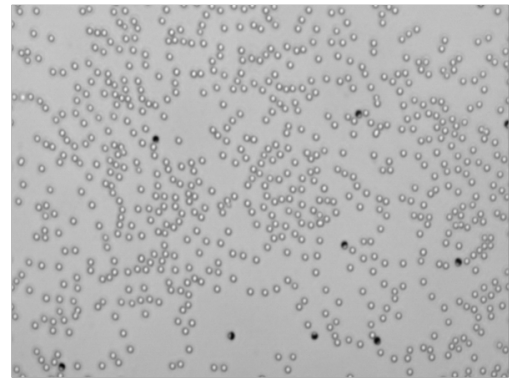

+5 mins

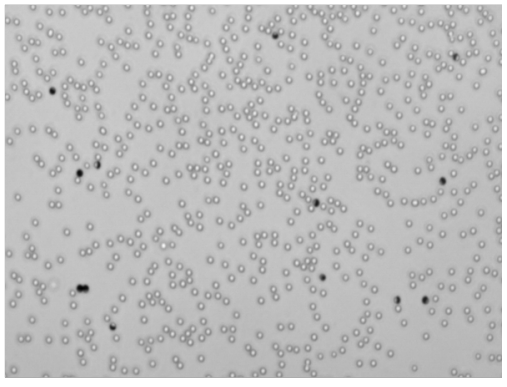

+10 mins

Figure 6: time dependence of interactions around copper Janus particles. 


\section{3 $\mathrm{Ag} @ \mathrm{SiO}_{2}$ Janus - on - off - repulsive interactions}
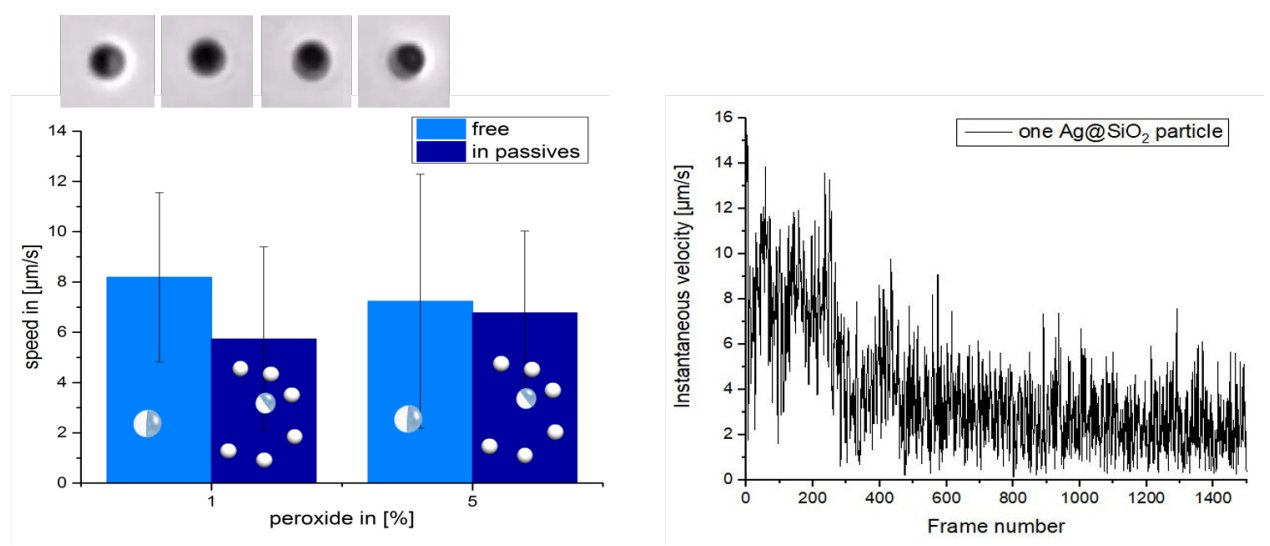

Figure 7: orientation and speed of silver catalyzed Janus particles.

As can be seen from the upper line of Figure 7, the silver Janus particle do not maintain a very stable orientation during their swimming. Different orientations of the cap, as well as small detachments from the substrate into z-direction are permanently noticeable. The swimming speed decays quickly due to the chemical interactions of silver with the peroxide (dissolution and passivation), limiting the life time of the silver Janus particles to maximum a few minutes, depending on the fuel concentration. Despite the fact that we have only considered a very short time span after the onset of motion, the higher peroxide concentration at $5 \%$ peroxide enhances the dissolution and passivation reactions happening on the silver surface, so that we do not see an increase in speed as expected.

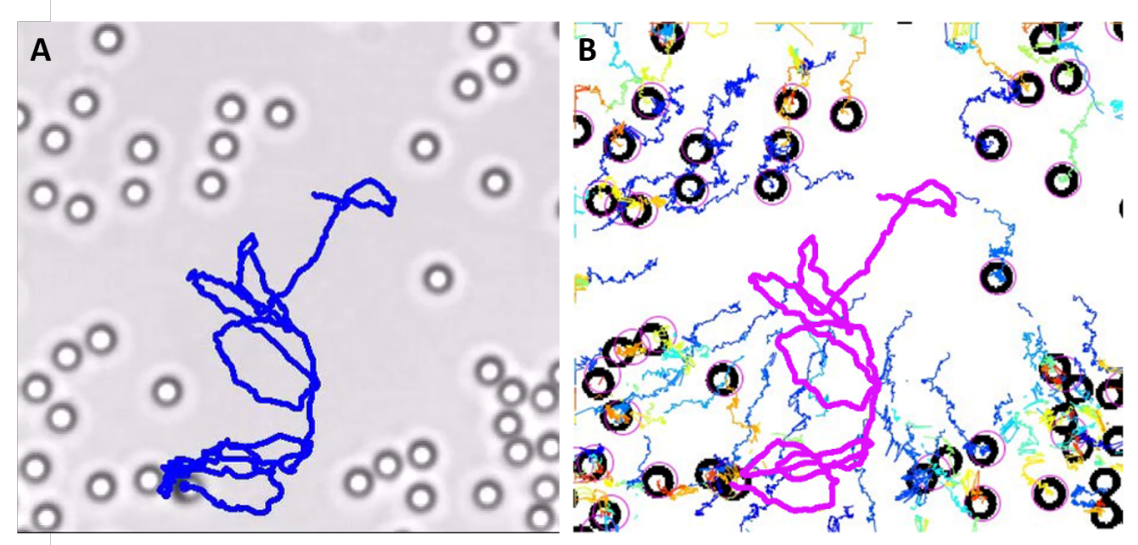

Figure 8: trajectory and interaction of silver Janus particles.

Looking at the swimming trajectory and the meanwhile interactions with the passive particles, 
an interesting observation peaks out: the repelling nature of the interactions seems to be strong only while the particles is swimming along the substrate. During the course of the particle motion, the particle frequently detaches from the substrate and in these phases, it can move very closely to the passive particles. After reaching the surface again, normally also characterized by a swimming vector close to 0 degrees, the interactions immediately become stronger. However, as mentioned previously, the life time of the silver Janus particles in peroxide is short, which is also the main reason for the short observability of the exclusion zones, see Figure 9 . While initially the exclusion zones can be very pronounced, after a few minutes most effects are already re-mediated by random Brownian motion of the passive particles. About 10 minute after peroxide addition, the Ag particles have become completely inactive and no exclusion zone is deducible after all.

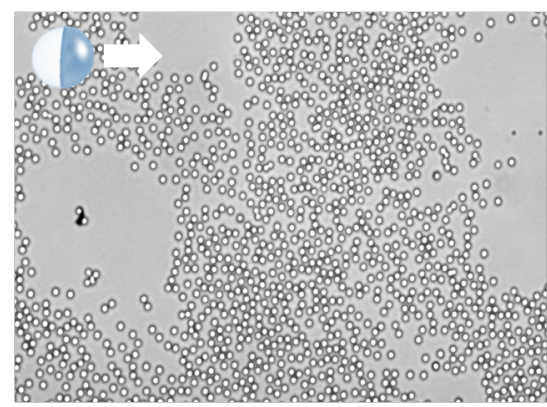

$1 \mathrm{~min}$ after peroxide addition

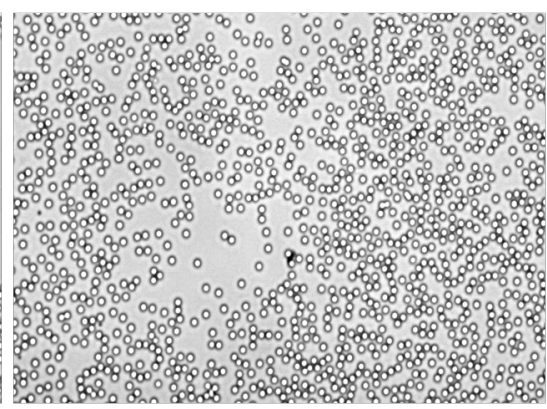

+5 mins

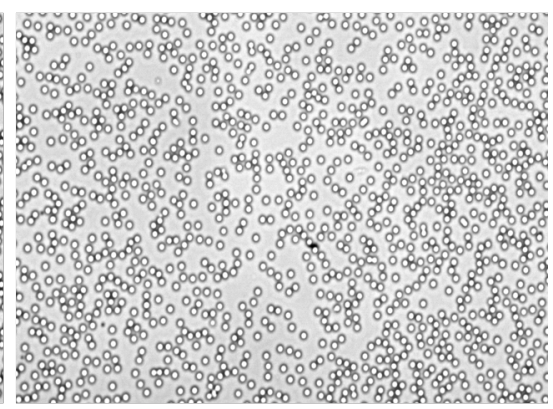

+10 mins

Figure 9: time dependence of interactions around silver Janus particles.

\section{Conclusions}

Similarly to our previous findings, where we demonstrated that we can tune the nature of interactions using different fuels and wave length for light driven swimmers, the interactions are also strongly influenced by the chosen peroxide degrading surface. The use of different swimmers in peroxide, all based on silica spheres, enables us to precisely evaluate the swimming direction of the Janus particles, resulting in different swimming directions. Platinum swimmers show a well oriented, away-from the cap motion, which is steady as long as the peroxide concentration is kept constant. The gradient produced by the particles is attractive for passive $\mathrm{SiO}_{2}$ particles, leading to raft formation. However, this interaction is short ranged and only experienced after direct contact. Differently, the copper Janus particles, which also present a constant motion vector parallel to the substrate, move towards the metal cap. The precise origin of this opposite movement direction is still under investigation, but the gradient produced by the particles leads to repulsive interactions with passive particles, forming exclusion zones around the passives. The most complex phenomenology is observed for silver particles, which often move with 0 degree motion vector parallel to the surface. However, Ag Janus particles are also able to turn away and even detach from the surface into z-direction. While the interactions with passives are clearly repulsive while the particles move in 0 degree orientation on the surface, when detached from the surface the interactions with passives show many different varieties. We assume, that controlling the orientation, one should be able 
to turn the phoretic interactions on and off, which is most likely due to a strong influence of the surface flows.

\section{Acknowledgements}

The authors thank the Volkswagen foundation for the Freigeist fellowship (grant number 91619), as well as the Kaercher foundation. Interesting comments and discussions on the interactions and the manuscript with Ivo Buttinoni, Benno Liebchen, Hartmut Loewen, Falko Schmidt, Ian Madden and Erik Luijten, as well as Mihail N. Popescu and Joost de Graaf. Linlin Wang is greatly acknowledged for critical proofreading of the manuscript.

\section{References}

[1] Eric Lauga, Willow R. DiLuzio, George M. Whitesides, and Howard A. Stone. Swimming in circles: Motion of bacteria near solid boundaries. Biophysical Journal, 90(2):400-412, 2006.

[2] Juliane Simmchen, Jaideep Katuri, W.E. William E. Uspal, M.N. Mihail N. M.N. Popescu, Mykola Tasinkevych, Samuel Sánchez, S. S?nchez, and Samuel Sánchez. Topographical pathways guide chemical microswimmers. Nature Communications, 7(May 2015):10598, 2016.

[3] W. E. Uspal, M. N. Popescu, S. Dietrich, and M. Tasinkevych. Self-propulsion of a catalytically active particle near a planar wall: from reflection to sliding and hovering. Soft Matter, 11(3):434-438, 2015.

[4] Sambeeta Das, Astha Garg, Andrew I Campbell, Jonathan Howse, Ayusman Sen, Darrell Velegol, Ramin Golestanian, and Stephen J Ebbens. Boundaries can steer active Janus spheres. Nature communications, 6:8999, 2015.

[5] C. Maggi, J. Simmchen, F. Saglimbeni, J. Katuri, M. Dipalo, F. De Angelis, S. Sanchez, and R. Di Leonardo. Self-Assembly of Micromachining Systems Powered by Janus Micromotors. Small, 12(4), 2016.

[6] Daisuke Takagi, Jérémie Palacci, Adam B. Braunschweig, Michael J. Shelley, and Jun Zhang. Hydrodynamic capture of microswimmers into sphere-bound orbits. Soft Matter, 10(11):1784-1789, 2014.

[7] Larysa Baraban, Denys Makarov, Robert Streubel, Ingolf Mönch, Daniel Grimm, Samuel Sanchez, and Oliver G Schmidt. Catalytic Janus Motors on Microfluidic Chip: Deterministic Motion for Targeted Cargo Delivery. ACS Nano, 6(4):3383-3389, apr 2012.

[8] L. Baraban, M. Tasinkevych, M. N. Popescu, S. Sanchez, S. Dietrich, and O. G. Schmidt. Transport of cargo by catalytic Janus micro-motors. Soft Matter, 8(1):48-52, 2012.

[9] Guanjia Zhao, Hong Wang, Samuel Sanchez, Oliver G. Schmidt, and Martin Pumera. Artificial microcinderella based on self-propelled micromagnets for the active separation of paramagnetic particles. Chemical Communications, 49(45):5147-5149, 2013.

[10] Gastón Miño, Thomas E. Mallouk, Thierry Darnige, Mauricio Hoyos, Jeremi Dauchet, Jocelyn Dunstan, Rodrigo Soto, Yang Wang, Annie Rousselet, and Eric Clement. Enhanced diffusion due to active swimmers at a solid surface. Physical Review Letters, 106(4):1-4, 2011.

[11] Andreas Zöttl and Julia M. Yeomans. Enhanced bacterial swimming speeds in macromolecular polymer solutions. Nature Physics, pages 1-16, 2019.

[12] Ivo Buttinoni, Julian Bialké, Felix Kümmel, Hartmut Löwen, Clemens Bechinger, and Thomas Speck. Dynamical clustering and phase separation in suspensions of self-propelled colloidal particles. Physical Review Letters, 110(23):1-5, 2013. 
[13] Felix Kümmel, Parmida Shabestari, Celia Lozano, Giovanni Volpe, and Clemens Bechinger. Formation, compression and surface melting of colloidal clusters by active particles. Soft Matter, 11(31):6187-6191, 2015 .

[14] Alicia Boymelgreen, Gilad Yossifon, and Touvia Miloh. Propulsion of Active Colloids by Self-Induced Field Gradients. Langmuir, 32(37):9540-9547, 2016.

[15] Alicia M. Boymelgreen, Tov Balli, Touvia Miloh, and Gilad Yossifon. Active colloids as mobile microelectrodes for unified label-free selective cargo transport. Nature Communications, 9(1):1-8, 2018.

[16] Ahmet F. Demirörs, Mehmet Tolga Akan, Erik Poloni, and André R. Studart. Active cargo transport with Janus colloidal shuttles using electric and magnetic fields. Soft Matter, 14(23):4741-4749, 2018.

[17] Ahmet F. Demirörs, Fritz Eichenseher, Martin J. Loessner, and André R. Studart. Colloidal shuttles for programmable cargo transport. Nature Communications, 8(1):1-7, 2017.

[18] Lina Zhang, Hui Zhang, Mei Liu, and Bin Dong. Reprogrammable Logic Gate and Logic Circuit Based on Multistimuli-Responsive Raspberry-like Micromotors. ACS Applied Materials and Interfaces, 8(24):15654-15660, 2016.

[19] Alicia M. Boymelgreen, Tov Balli, Touvia Miloh, and Gilad Yossifon. Active colloids as mobile microelectrodes for unified label-free selective cargo transport. Nature Communications, 9(1):1-8, 2018.

[20] Linlin Wang, Mihail N. Popescu, Fernando Stavale, Astrid Ali, Thomas Gemming, and Juliane Simmchen. Cu@TiO2 Janus microswimmers with a versatile motion mechanism. Soft Matter, 14(34):69696973, 2018.

[21] Dhruv P. Singh, Udit Choudhury, Peer Fischer, and Andrew G. Mark. Non-Equilibrium Assembly of Light-Activated Colloidal Mixtures. Advanced Materials, 29(32):1-7, 2017.

[22] Jie Zhang, Erik Luijten, Bartosz A. Grzybowski, and Steve Granick. Active colloids with collective mobility status and research opportunities. Chemical Society Reviews, 46(18):5551-5569, 2017.

[23] Ran Niu and Thomas Palberg. Modular approach to microswimming. Soft Matter, 14(37):7554-7568, 2018.

[24] Eva Kanso and Sébastien Michelin. Phoretic and hydrodynamic interactions of weakly confined autophoretic particles. Journal of Chemical Physics, 150(4), 2019.

[25] M. N. Popescu, W. E. Uspal, Z. Eskandari, M. Tasinkevych, and S. Dietrich. Effective squirmer models for self-phoretic chemically active spherical colloids. European Physical Journal E, 41(12), 2018.

[26] Bryan Robertson, Mu Jie Huang, Jiang Xing Chen, and Raymond Kapral. Synthetic Nanomotors: Working Together through Chemistry. Accounts of Chemical Research, 51(10):2355-2364, 2018.

[27] Benno Liebchen, Davide Marenduzzo, and Michael E. Cates. Phoretic Interactions Generically Induce Dynamic Clusters and Wave Patterns in Active Colloids. Physical Review Letters, 118(26):1-5, 2017.

[28] B. Liebchen and H. Löwen. Which Interactions Dominate in Active Colloids? 061102(February), 2018.

[29] Joost De Graaf and Joakim Stenhammar. Lattice-Boltzmann simulations of microswimmer-tracer interactions. Physical Review E, 95(2):1-10, 2017.

[30] Raphael Wittkowski, Joakim Stenhammar, and Michael E Cates. Nonequilibrium dynamics of mixtures of active and passive colloidal particles Related content Active cluster crystals. 2017.

[31] Julian Stürmer, Maximilian Seyrich, and Holger Stark. Chemotaxis in a binary mixture of active and passive particles. Journal of Chemical Physics, 150(21), 2019.

[32] Jonathan R. Howse, Richard A L Jones, Anthony J. Ryan, Tim Gough, Reza Vafabakhsh, and Ramin Golestanian. Self-Motile Colloidal Particles: From Directed Propulsion to Random Walk. Physical Review Letters, 99(4):8-11, 2007.

[33] J. Simmchen, V. Magdanz, S. Sanchez, S. Chokmaviroj, D. Ruiz-Molina, A. Baeza, and O.G. Schmidt. Effect of surfactants on the performance of tubular and spherical micromotors-a comparative study. RSC Advances, 4(39), 2014. 